\section{Treinamento aeróbico e de força no tratamento do diabetes gestacional: uma revisão sistemática}

\section{Influence of aerobic and strength training in the gestational diabetes treatment: a systematic review}

Elisa Gouvêa Portella'

Roberta Bgeginski $i^{1,2}$

Luiz Fernando Martins Kruel ${ }^{1}$

\section{RESUMO}

O diabetes mellitus gestacional (DMG) é uma complicação comum cuja prevalência tem crescido constantemente. Evidências sugerem que intervenções em gestantes com DMG podem reduzir a ocorrência de desfechos adversos. Apesar de o exercício físico ser bem aceito como uma terapia complementar no manejo do diabetes tipo 2 fora da gestação, no DMG ainda não se sabe qual a melhor prescrição para obter os melhores resultados. Portanto, este estudo teve como objetivo revisar as evidências científicas sobre o efeito de diferentes tipos de treinamento físico no tratamento do DMG. Foram consultadas as bases de dados, Scopus, Pubmed e Scielo. Foram excluídos os estudos de revisão sistemática, editoriais, cartas ao editor, relatos de caso, estudos laboratoriais e artigos que não avaliassem dos efeitos de intervenção através de exercício aeróbico ou de força, em mulheres diagnosticadas com DMG. Foram identificados 661 estudos e incluídos 6 na análise final. Foram encontrados estudos que utilizaram o treinamento físico como parte do tratamento do DMG, a maioria demonstrou efeito benéfico, como melhora do controle glicêmico e diminuição das necessidades de insulina. O tipo de exercício escolhido pela maioria dos pesquisadores foi o aeróbico, entretanto os estudos que utilizaram o exercício de força também demonstraram melhor controle glicêmico. Considerando os achados desses estudos, o treinamento físico tanto aeróbico como de força, apresentou efeitos benéficos para mulheres com DMG, uma vez que ajudou a controlar a glicemia e reduzir as necessidades de insulina.

\section{PALAVRAS-CHAVE}

Gestação; Resistência à insulina; Exercício.

\begin{abstract}
The gestational diabetes mellitus (GDM) is a common complication whose prevalence has steadily grown. Evidence suggests that interventions in pregnant women with GDM may reduce the occurrence of adverse outcomes. Although exercise is well accepted as a complementary therapy in type 2 diabetes in non-pregnant women, but is still unclear what is the best prescription for best results in the management of GDM. Therefore, this study aimed to review the scientific evidence on the effect of different types of exercise training in the treatment of GDM. Databases such as Scopus, Pubmed e Scielo were consulted. Systematic reviews, editorials, letters to the editor, case reports, laboratory studies and articles that do not evaluated the effects of intervention through aerobic or strength exercise in women diagnosed with GDM were excluded. 661 studies were first identified and 6 were included in the final analysis. Studies using exercise as part of GDM treatment demonstrated beneficial effects, such as improved glycemic control and decreased insulin requirements. The type of exercise chosen by most researchers was aerobic, however studies using strength exercise also demonstrated to improve glycemic control. The studies founded in the present systematic review, showed beneficial effects of aerobic and strength training for women with GDM, once belped to control blood sugar and to reduce insulin requirements.
\end{abstract}

\section{KEYWORDS}

Pregnancy; Insulin resistance; Exercise.
Rev Bras Ativ Fis Saúde p. 400-409 DOI

http://dx.doi.org/10.12820/rbafs.v.19n4p400

1 Universidade Federal do Rio Grande do Sul, Escola de Educação Física, Laboratório de Pesquisa do Exercício, Grupo de Pesquisa em Atividades Aquáticas e Terrestres, Porto Alegre, Rio Grande do Sul, Brasil.

2 Universidade Federal do Rio Grande do Sul, Programa de Pós-graduacão em Medicina: Ciências Médicas, Porto Alegre, Rio Grande do Sul, Brasil. 


\section{INTRODUÇÃO}

O diabetes mellitus gestacional (DMG) é definido como qualquer grau de intolerância à glicose primeiramente diagnosticado durante a gestação ${ }^{1-3}$. É uma complicação comum cuja prevalência tem crescido constantemente ${ }^{4,5}$, podendo variar de 1 a $14 \%{ }^{2}$, dependendo da população estudada e do critério diagnóstico utilizado ${ }^{1}$. Com relação às populações de maior risco, estão latinos, negros, americanos nativos, indígenas australianos (aborígenes), sul-asiáticos, habitantes das ilhas do Pacífico ${ }^{6}$.

A presença do DMG leva a desfechos adversos tanto para o feto quanto para a mãe, sendo a complicação mais comum a macrossomia fetal, definido como peso ao nascer acima de $4000 \mathrm{~g}$, quando $95 \%$ da idade gestacional for alcançada ${ }^{1,7}$, o que pode levar a dificuldades durante o parto ${ }^{8}$, ocasionando traumas ao recém nascido ${ }^{7}$. Ao longo da vida da criança há aumento do risco de desenvolver intolerância à glicose e obesidade, assim como há uma maior chance da mãe necessitar de cesárea e de desenvolver desordens hipertensivas e diabetes tipo 2 após o parto ${ }^{1,9}$.

O tratamento inicial do DMG envolve modificações na dieta e monitoramento da glicose ${ }^{10}$, no entanto, quando o controle glicêmico não é alcançado por meio desse tipo de tratamento, torna-se necessário o uso de insulina ou antidiabéticos orais ${ }^{11,12}$. Uma vez que o uso de medicação resolve a hiperglicemia sem afetar suas causas, como a resistência à insulina, o exercício parece ser uma intervenção não-farmacológica mais coerente, pois além de melhorar a sensibilidade à insulina e estimular o músculo a captar glicose sem a presença desse hormônio, promove mudanças na composição corporal, ajuda a limitar o ganho de peso, melhora o condicionamento cardiovascular, provoca mudanças nas adiponectinas, reduz o estresse oxidativo, melhora a qualidade de vida e promove mudanças bioquímicas, fisiológicas e morfológicas no músculo esquelético ${ }^{13,14}$.

A maior parte dos benefícios do treinamento físico, tanto aeróbico como de força, para indivíduos com diabetes tipo 2, estão relacionados com melhorias agudas e crônicas na ação da insulina ${ }^{15,16}$. O DMG e o diabetes tipo 2 são bastante similares, pois seus mecanismos estão centrados no aumento da resistência à insulina e, consequentemente, na diminuição da tolerância à glicose. Devido a esse fato, os mecanismos pelos quais o exercício induz benefícios no diabetes tipo 2 podem ser explorados no $\mathrm{DMG}^{14}$.

Tanto o treinamento aeróbico, quanto o de força são recomendados pelas diretrizes internacionais para mulheres com $\mathrm{DMG}^{1-17}$ como uma terapia complementar e não-farmacológica, uma vez que a inatividade física e a vida sedentária podem colocar a mãe e o feto em risco de doenças através das adaptações gestacionais ${ }^{8}$. Além disso, a prática de exercício durante a gestação também está relacionado com a prevenção da hipertensão e do diabetes tipo 2 pós-gestação, visto que ajuda a evitar o ganho excessivo de peso ${ }^{18}$. Porém, ainda não foi determinado qual a frequência, intensidade, duração e tipo de atividade são necessárias para fornecer os melhores resultados para mulheres com DMG.

Portanto, este estudo tem como objetivo revisar sistematicamente as evidências sobre o efeito de diferentes tipos de treinamento físico no tratamento do DMG, e através dessas evidências, proporcionar aos profissionais da área 
da saúde informações que possam dar-lhes segurança para indicar a prática de exercício físico e auxiliar os profissionais da educação física a desenvolverem uma prescrição de treinamento segura e adequada para gestantes com DMG.

\section{MÉTODOS}

Para realização da presente revisão sistemática, foram utilizadas referências clássicas e atuais sobre o referido tema. $\mathrm{O}$ método incluiu estratégia de busca, critérios de inclusão, critérios de exclusão, identificação dos estudos e extração de dados. As bases de dados utilizadas foram Scopus, Pubmed e Scielo; os estudos deveriam ser publicados em língua portuguesa, espanhola e inglesa, sem limite de data, conduzidos em humanos, além de apresentarem os resultados de efeitos crônicos de treinamento (aeróbico ou de força). Para tal busca, palavras-chave como diabetes gestational, pregnancy, insulin resistance, exercise, physical activity e suas respectivas traduções foram combinadas. A fim de localizar possíveis artigos que não fossem encontrados na pesquisa inicial, foram consultadas as referências bibliográficas dos artigos identificados. Foram excluídos os estudos de revisão sistemática, editoriais, cartas ao editor, relatos de caso, estudos laboratoriais e artigos que não reportassem a avaliação dos efeitos de intervenção através de exercício aeróbico ou de força, em mulheres previamente diagnosticadas com DMG.

Os estudos foram selecionados por dois pesquisadores independentes. Foi realizada a leitura dos títulos para verificar se atendiam aos objetivos do estudo. Em seguida, foram excluídos os artigos em duplicata e foi realizada a leitura dos resumos dos artigos para verificar se atendiam aos critérios de inclusão do presente estudo. Os estudos que atenderam aos critérios de inclusão foram recuperados para leitura do texto completo e nova avaliação quanto aos critérios de inclusão. Por fim, os artigos selecionados foram lidos na íntegra e posteriormente analisados para a extração dos dados. O fluxograma do processo de busca e seleção dos artigos encontrados está apresentado na figura 1 .

FIGURA 1 - Flowchart da busca nas bases de dados.
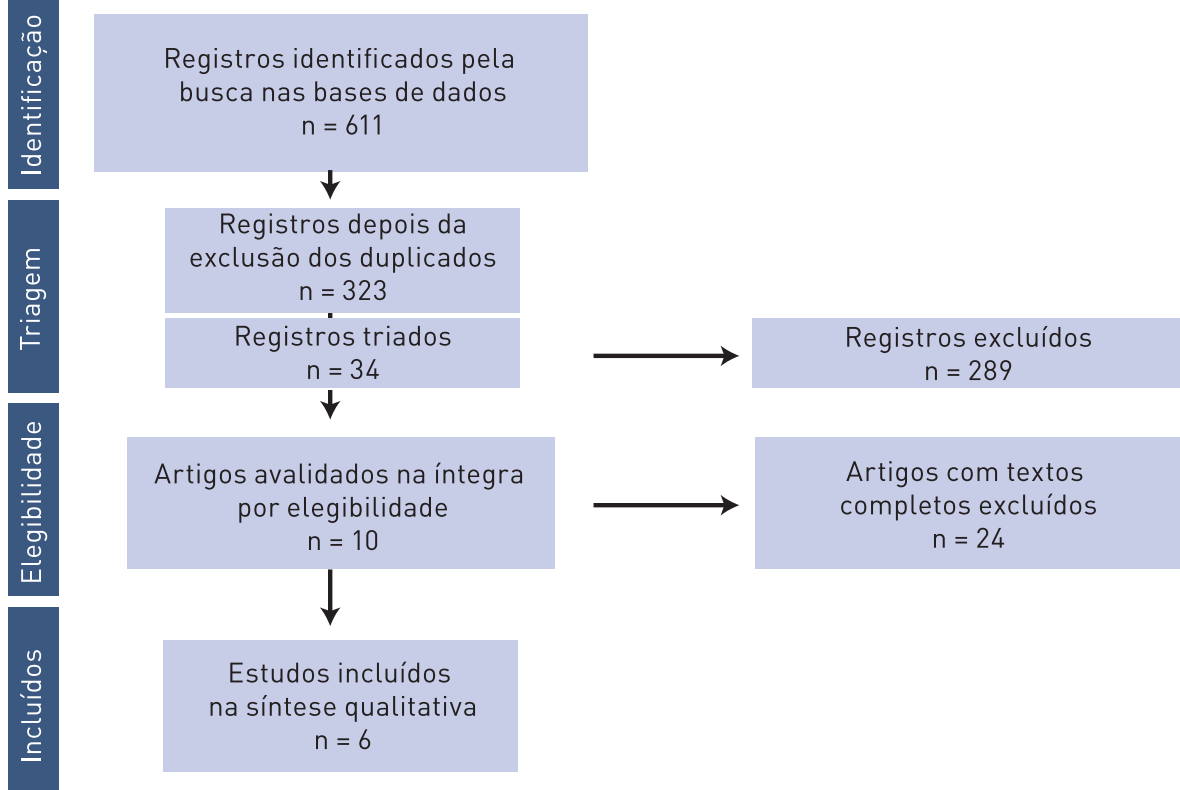

Artigos avalidados na íntegra por elegibilidade $n=10$

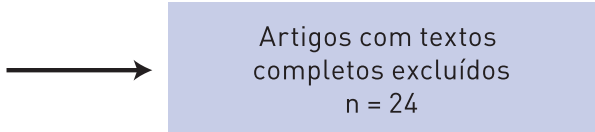

Estudos incluídos

na síntese qualitativa $n=6$ 


\section{RESULTADOS}

Após verificação nas diferentes bases de dados, foram localizados 661 estudos que apresentaram relação com a temática pesquisada e os descritores utilizados. Foram excluídos os estudos em duplicada, restando 323 estudos. Destes, 313 foram excluídos após a revisão dos títulos, resumos e palavras-chave, além da utilização dos critérios de inclusão e exclusão, restando 10 estudos que foram lidos na íntegra pelos dois pesquisadores. Foram incluídos seis estudos ${ }^{19-24}$ na análise qualitativa final (Figura 1).

Os estudos selecionados foram publicados entre 1989 e 2012. A Tabela 1 apresenta um resumo da amostra, do protocolo de exercício e os resultados

TABELA 1 - Efeitos do treinamento aeróbico no controle glicêmico e necessidade de insulina em gestantes com DMG.

\begin{tabular}{|c|c|c|c|c|c|}
\hline AUTOR & OBJETIVO & AMOSTRA & INTERVENC̣ÃO & RESULTADO & CONCLUSÃO \\
\hline $\begin{array}{l}\text { Jovanovic- } \\
\text { Peterson } \\
\text { et al. }{ }^{.9}\end{array}$ & $\begin{array}{l}\text { Avaliar o impacto } \\
\text { de um programa } \\
\text { de treinamento } \\
\text { em ergômetro de } \\
\text { braço, que não está } \\
\text { associado com } \\
\text { atividade uterina e } \\
\text { bradicardia fetal, } \\
\text { na tolerância } \\
\text { a glicose em } \\
\text { gestantes com } \\
\text { DMG. }\end{array}$ & $\begin{array}{l}\text { Grupo I= dieta (n } \\
=9) \\
\text { Grupo II= } \\
\text { dieta+exercício } \\
(n=10)\end{array}$ & $\begin{array}{l}\text { Nutrição: dieta } 24-30 \mathrm{kcal} / \\
\mathrm{kg} / 24 \mathrm{~h} \text {. } \\
40 \% \text { carboidrato, } 20 \% \\
\text { proteína e } 40 \% \text { gordura. } \\
\text { Exercício: supervisionado } \\
\text { em cicloergômetro de } \\
\text { braço; } 3 \times / \mathrm{sem} ; \text { intensidade } \\
\text { de } 50 \% \mathrm{VO}_{2 \text { max }} ; 20 \mathrm{~min} / \\
\text { sessão; } 6 \text { sem. }\end{array}$ & 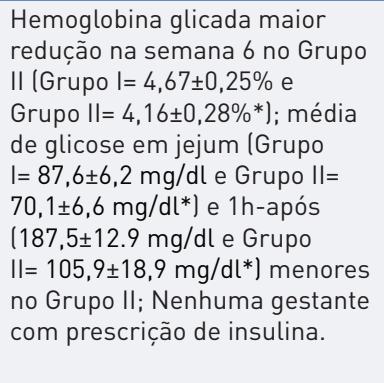 & $\begin{array}{l}\text { Um programa de } \\
\text { treinamento em } \\
\text { ergômetro de braço } \\
\text { pode ser uma opção } \\
\text { de tratamento útil } \\
\text { para mulheres com } \\
\text { DMG e, pode prevenir } \\
\text { o tratamento com } \\
\text { insulina. }\end{array}$ \\
\hline $\begin{array}{l}\text { Bung et } \\
\text { al. }^{20}\end{array}$ & $\begin{array}{l}\text { Investigar a } \\
\text { eficácia de um } \\
\text { programa de } \\
\text { exercício na } \\
\text { normalização } \\
\text { da tolerância a } \\
\text { glicose. }\end{array}$ & $\begin{array}{l}\text { Dieta+Exercício } \\
(D+E): n=17(I G: \\
30,3 \pm 1,9 \text { sem); } \\
\text { Dieta+Insulina } \\
(D+I): \\
n=17(I G: 30,3 \pm 2,0 \\
\text { sem) }\end{array}$ & $\begin{array}{l}\text { Nutrição: dieta } 30 \mathrm{kcal} / \mathrm{kg} / \\
\text { dia. } \\
\text { Exercício: supervisionado } \\
\text { em bicicleta estacionária; } \\
3 \times / \text { sem; intensidade de } \\
50 \% \mathrm{VO}_{2 \text { máx }} ; 5 \text { min/sessão; } \\
\text { treinamento do diagnóstico } \\
\text { da DMG até o parto. }\end{array}$ & $\begin{array}{l}\text { Não houve diferença } \\
\text { significativa entre os grupos } \\
\text { nas determinações de glicose } \\
\text { sanguínea semanais }(D+E=5,2 \\
\pm 0,30 \mathrm{mmol} / \mathrm{L} \text { e } \mathrm{D}+\mathrm{I}=4,92 \pm 0,35 \\
\mathrm{mmol} / \mathrm{L} \text { ); Controle glicêmico } \\
\text { por meio do exercício foi efetivo. }\end{array}$ & $\begin{array}{l}\text { O programa de } \\
\text { exercício descrito } \\
\text { parece ser seguro } \\
\text { e, pode servir como } \\
\text { modelo para prescrição } \\
\text { de exercício para } \\
\text { gestantes diabéticas } \\
\text { para atingir uma } \\
\text { melhora da tolerância a } \\
\text { insulina. }\end{array}$ \\
\hline $\begin{array}{l}\text { Avery et } \\
\text { al. }{ }^{21}\end{array}$ & $\begin{array}{l}\text { Examinar a } \\
\text { efetividade de } \\
\text { um programa de } \\
\text { exercício aeróbico } \\
\text { de moderada } \\
\text { intensidade } \\
\text { realizado } \\
\text { basicamente em } \\
\text { casa por mulheres } \\
\text { com DMG. }\end{array}$ & $\begin{array}{l}\text { Exercício (E): } n=15 \\
\text { (IG: } 28,7 \pm 3,0 \text { sem) } \\
\text { Controle (C): } n=14 \\
\text { (IG: } 26,3 \pm 8,1 \text { sem) }\end{array}$ & $\begin{array}{l}\text { Nutrição: dieta padrão para } \\
\text { DMG. } \\
\text { Exercício: parcialmente } \\
\text { supervisionado em bicicleta } \\
\text { ergométrica ou caminhada; } \\
3-4 x / \text { sem ( } 2 x / \text { sem sem } \\
\text { supervisão e } 2 x / s e m \text { com } \\
\text { supervisão); } 30 \mathrm{~min} / \mathrm{sessão} \\
\text { intensidade de } 70 \% \text { da } \mathrm{FC} \text { máx } \\
\text { (220- idade); treinamento da } \\
34^{\mathrm{a}} \text { sem até o parto. }\end{array}$ & $\begin{array}{l}\text { Hemoglobina glicada sem } \\
\text { diferença significativa entre os } \\
\text { grupos ( } E=5,33 \% \text { e } C=5,25 \%) \\
\text { Uso de insulina não diferiu } \\
\text { entre os grupos. }\end{array}$ & $\begin{array}{l}\text { O programa de } \\
\text { exercícios não foi capaz } \\
\text { de reduzir os níveis de } \\
\text { glicose sanguínea, mas } \\
\text { resultou em modesta } \\
\text { melhora da capacidade } \\
\text { cardiorrespiratória. }\end{array}$ \\
\hline $\begin{array}{l}\text { Artal et } \\
\text { al. }^{22}\end{array}$ & $\begin{array}{l}\text { Avaliar se uma } \\
\text { dieta com restrição } \\
\text { ao ganho de peso, } \\
\text { aliada ou não ao } \\
\text { exercício, é capaz } \\
\text { de gerar impacto } \\
\text { no controle } \\
\text { glicêmico, nos } \\
\text { desfechos da } \\
\text { gestação e no } \\
\text { ganho total de } \\
\text { peso em mulheres } \\
\text { obesas com DMG. }\end{array}$ & $\begin{array}{l}\text { Dieta (D): } n=57 \\
\text { (IG: } 28,0 \pm 5,1 \text { sem) } \\
\text { Dieta+exercício } \\
(D+E): n=39 \text { (IG: } \\
29,4 \pm 4,9 \text { sem) }\end{array}$ & $\begin{array}{l}\text { Nutrição: dieta padronizada } \\
\text { para DMG, } 40-45 \% \text { de } \\
\text { carboidrato. } \\
\text { Exercício: parcialmente } \\
\text { supervisionado em esteira } \\
\text { ou bicicleta estacionária; } \\
7 x / \text { sem (1x/sem com } \\
\text { supervisão } \\
6 x / \text { sem sem supervisão); } \\
\text { intensidade de } 60 \% \mathrm{VO}_{2} \\
\text { máx } 20 \text { min/sessão; } \\
\text { Treinamento da } 33^{\mathrm{a}} \text { sem de } \\
\text { gestação até o parto. }\end{array}$ & $\begin{array}{l}\text { Diferença significativa no } \\
\text { ganho de peso ( } D+E=53 \% \text { e } D= \\
78,9 \% * * \text {. } \\
\text { Sem diferença significativa } \\
\text { entre os grupos na necessidade } \\
\text { de insulina. ( } D+E \text { : } n=13 \text { e } D \text { : } \\
n=22) \text {. }\end{array}$ & $\begin{array}{l}\text { Um programa de } \\
\text { restrição calórica e } \\
\text { exercício realizado por } \\
\text { mulheres obesas com } \\
\text { DMG, resultou em, } \\
\text { ganho de peso limitado, } \\
\text { menor número } \\
\text { macrossomia, e } \\
\text { nenhum efeito adverso } \\
\text { a gestação. }\end{array}$ \\
\hline
\end{tabular}

IG: idade gestacional; DMG: Diabetes Mellitus Gestacional; $\mathrm{VO}_{2 \text { máx }}$ : Consumo Máximo de Oxigênio; $\mathrm{FC}_{\text {Max }}$ : Frequência Cardíaca Máxima; ${ }^{*} p<0,001 ;{ }^{* *} p<0,01$ 
dos quatro estudos ${ }^{19-22}$ encontrados na presente revisão e que objetivaram analisar o efeito do treinamento aeróbico no tratamento do DMG e a Tabela 2 apresenta as mesmas características anteriormente citadas dos dois ${ }^{23,24}$ estudos encontrados e que objetivaram analisar o efeito do treinamento de força no tratamento do DMG. As amostras dos estudos foram compostas de nove a 57 participantes, saudáveis e/ou com diagnóstico de DMG.

No que diz respeito ao efeito do treinamento aeróbico no controle glicêmico do DMG (Tabela 2), três estudos ${ }^{20-22}$ optaram pelo exercício em bicicleta ergométrica. Dos quatro estudo selecionados em três deles, o exercício foi realizado três vezes por semana e, em um estudo, ele foi realizado sete vezes por semana, sendo que as sessões variaram de 20 a 45 minutos. Em dois estudos, a intensidade foi de $50 \%$ do consumo máximo de oxigênio $\left(\mathrm{VO}_{2 \max }\right)$ e, nos outros dois, foi de $60 \% \mathrm{VO}_{2 \max }$ e $70 \% \mathrm{VO}_{2 \max }$. A duração dos programas dos estudos foi de no mínimo seis semanas.

Dois estudos ${ }^{19,21}$ avaliaram a hemoglobina glicada e encontraram resultados diferentes. Jovanovic-Peterson et al. ${ }^{19}$, encontraram menores valores percentuais dessa variável para o grupo tratado com dieta e exercício (pré intervenção: $4,83 \% \pm 0,33 \%$; pós intervenção: 4,16\% $\pm 0,28 \%$ ), em comparação ao grupo tratado somente com dieta (pré intervenção: 4,86\% $\pm 0,39 \%$; pós intervenção: $4,67 \% \pm 0,25 \% ; \mathrm{p}<0,001)$. Avery et al. ${ }^{21}$ não encontraram diferença significativa entre os grupos dieta padrão e exercício. Em relação ao uso de insulina, três estudos ${ }^{19,21,22}$ relataram não haver diferença entre os grupos intervenção e controle para esta variável.

Em relação ao efeito do treinamento de força no controle glicêmico (Tabela 2), apenas dois estudos ${ }^{23,24}$ foram encontraram e relataram que a exigência de insulina foi menor no grupo intervenção, comparada ao grupo controle,

TABELA 2 - Efeitos do treinamento de força no controle glicêmico e necessidade de insulina em gestantes com DMG.

\begin{tabular}{|c|c|c|c|c|c|}
\hline AUTOR & OBJETIVO & AMOSTRA & INTERVENÇÃO & RESULTADO & CONCLUSÃO \\
\hline $\begin{array}{l}\text { Brankston } \\
\text { et al. } .^{23}\end{array}$ & $\begin{array}{l}\text { Examinar os } \\
\text { efeitos de um } \\
\text { treinamento de força, } \\
\text { do tipo circuito, } \\
\text { na necessidade } \\
\text { de insulina em } \\
\text { mulheres com DMG. }\end{array}$ & $\begin{array}{l}\text { Dieta (D): } n=16 \\
\text { (IG: } 29,6 \pm 2,1 \\
\text { sem) } \\
\text { Dieta+Exercício } \\
\text { (D+E): } \\
n=16 \text { (IG: } \\
29,0 \pm 2,0 \text { sem) }\end{array}$ & $\begin{array}{l}\text { Nutrição: Dieta com } 40 \% \\
\text { carboidrato, } 20 \% \text { proteína } \\
\text { e } 40 \% \text { gordura. } \\
\text { Exercício: em forma de } \\
\text { circuito com banda elástica } \\
\text { não-supervisionado; } 3 \times / \text { sem; } \\
\text { intensidade moderada controlada } \\
\text { pela FC < } 140 \text { bpm e pela Escala } \\
\text { de Borg "um pouco intenso"; } \\
\text { treinamento com início entre } \\
26^{\mathrm{a}}-32^{\mathrm{a}} \text { até o parto. }\end{array}$ & $\begin{array}{l}\text { Sem diferença entre os } \\
\text { grupos no percentual de } \\
\text { sujeitos que necessitaram } \\
\text { de insulina ( } D=56,3 \% \text { e } \\
D+E=43,8 \% \text { ); Quantidade } \\
\text { de insulina prescrita foi } \\
\text { menor no } D+E \text { ( } D+E= \\
0,22 \pm 0,2 \text { U } / \mathrm{kg} \text { e } D=0,48 \pm 0,3 \\
\text { U/kg*); Tempo de latência } \\
\text { entre a primeira visita e o } \\
\text { início do uso de insulina foi } \\
\text { maior no grupo } D+E(D+E= \\
3,71 \pm 3,1 \text { sem e } D=1,11 \pm 0,8 \\
\text { sem*). }\end{array}$ & $\begin{array}{l}\text { O treinamento } \\
\text { de força pode } \\
\text { ajudar a evitar } \\
\text { o uso de terapia } \\
\text { com insulina para } \\
\text { mulheres acima do } \\
\text { peso com DMG. }\end{array}$ \\
\hline $\begin{array}{l}\text { Barros et } \\
\text { al. }{ }^{24}\end{array}$ & $\begin{array}{l}\text { Avaliar os efeitos } \\
\text { de um programa de } \\
\text { exercício de força, } \\
\text { com banda elástica, } \\
\text { na necessidade de } \\
\text { uso de insulina e } \\
\text { controle glicêmico } \\
\text { em pacientes com } \\
\text { DMG. }\end{array}$ & $\begin{array}{l}\text { Grupo exercício } \\
\text { (GE): } n=32 \text { (IG: } \\
31,56 \pm 2,29 \text { sem) } \\
\text { Grupo Controle } \\
\text { (GC): } n=32 \\
\text { (IG: } 31,06 \pm 2,30 \\
\text { sem) }\end{array}$ & $\begin{array}{l}\text { Nutrição: dieta de } 35 \mathrm{kcal} / \mathrm{kg} / \\
\text { dia, no último trimestre foram } \\
\text { adicionadas } 300 \mathrm{kcal} / \mathrm{dia} \text {. } \\
\text { Exercício: em forma de circuito } \\
\text { utilizando a banda elástica } \\
\text { parcialmente supervisionado } \\
\text { (1x/sem com supervisão e } 2 \mathrm{x} / \\
\text { sem sem supervisão); intensidade } \\
\text { controlada pela Escala de Borg, } \\
\text { "um pouco intenso"; treinamento } \\
\text { com início em } 24 \text { semanas até o } \\
\text { parto. }\end{array}$ & $\begin{array}{l}\text { Exigência de insulina } \\
\text { menor no } G E(E=56,3 \% \text { e } \\
G C=21,9 \% * *) \text {; Percentual } \\
\text { de semanas dentro da } \\
\text { faixa-alvo de glicose maior } \\
\text { no } G E(G C=0,41 \pm 0,31 \text { sem } \\
\left.\text { e } G E=0,63 \pm 0,30 \text { sem }^{* *}\right) \text {. }\end{array}$ & $\begin{array}{l}\text { O programa de } \\
\text { exercícios de } \\
\text { força foi efetivo, } \\
\text { reduziu o número } \\
\text { de pacientes que } \\
\text { necessitaram } \\
\text { de insulina e } \\
\text { melhoraram } \\
\text { o controle da } \\
\text { glicemia capilar } \\
\text { nessa população. }\end{array}$ \\
\hline
\end{tabular}


com gestantes que participaram do estudo entre $26^{a}-32^{a}$ semanas de gestação até o parto $(\mathrm{p}<0,05)$. Ambos os estudos realizaram intervenções com exercício do tipo circuito, utilizando banda elástica, com três sessões semanais de 40 minutos, e intensidade controlada pela Escala de Percepção de Esforço de $\mathrm{BORG}^{25}$.

\section{DISCUSSÃO}

\section{Efeito crônico do treinamento aeróbico e o DMG}

O treinamento do tipo aeróbico foi escolhido por alguns autores como estratégia para controlar a glicemia de gestantes com DMG, como apresentado na presente revisão. Bung et al. ${ }^{20}$, demonstraram em seu estudo que o programa de treinamento físico realizado por gestantes com DMG parece ter evitado o uso de insulina, visto que as gestantes do grupo exercício conseguiram manter sua glicemia normal, sem o uso de medicação. Eles atribuíram esse fato à capacidade do treinamento físico melhorar a sensibilidade à insulina, de maneira sustentada e de melhorar a captação da glicose sanguínea. Relataram também que, durante a prática do exercício físico, os hormônios contrarregulatórios da glicose (epinefrina, norepinefrina, glucagon, cortisol e hormônio do crescimento) possuem o importante papel de manter a sua homeostase. Além disso, imediatamente após o exercício físico, a captação da glicose pelos músculos continua, com o objetivo de restaurar as reservas de glicogênio. Desta forma, gestantes com DMG conseguiram aumentar o uso da glicose, reduzindo a hiperglicemia.

Esses dados corroboram as indicações da American Diabetes Association, que cita que "mulheres sem contraindicações clínicas e obstétricas são encorajadas a iniciar ou continuar, um programa de exercícios moderado, como parte do tratamento do $D M G$ ". Da mesma forma, a Canadian Diabetes Association sugeriu que "a atividade física deve ser encorajada, com sua frequência, tipo, duração e intensidade sendo adequados para cada gestante em função dos riscos obstétricos individuais" 18 .

Em gestantes saudáveis a secreção de insulina aumenta de duas a quatro vezes, com o objetivo de manter os níveis de glicose normais, o que não ocorre com as gestantes com DMG, visto que elas não conseguem aumentar a produção de insulina de maneira suficiente a compensar sua resistência ${ }^{26}$. O mecanismo pelo qual a gestação induz a resistência à insulina ainda não está claro, entretanto, a maioria das mulheres com DMG parecem apresentar disfunção nas células $\beta$-pancreáticas, que ocorre devido a uma resistência crônica à insulina presente antes da gestação $0^{27,28}$. Por outro lado, um número menor de mulheres parece apresentar essa comorbidade, devido a uma reação autoimune, que ocorre através de anticorpos ou antígenos para as células $\beta$-pancreáticas, que agem contra as ilhotas pancreáticas levando a sua destruição ${ }^{29}$.

Existe uma associação entre o DMG e a obesidade, como descrito em uma meta-análise que estimou os riscos de desenvolver DMG em duas, quatro e oito vezes maiores, entre mulheres com sobrepeso, obesas e severamente obesas, respectivamente ${ }^{30}$. Portanto, os exercícios sem sustentação de peso corporal, como em bicicleta estacionária, utilizado no estudo supracitado ${ }^{20}$ parecem ser ideais para gestante com DMG, previamente sedentárias, com sobrepeso ou obesas, pois as chances de provocar lesões músculo esqueléticas são menores, além de serem melhor tolerados por indivíduos sedentários ${ }^{13}$. 
Outro estudo ${ }^{19}$ objetivou investigar os efeitos do exercício físico sem sustentação de peso, em cicloergômetro de braço, e mesmo que esse envolva grupos musculares menores, foi capaz de melhorar o controle glicêmico nas mulheres com DMG, uma vez que diminuiu os níveis de glicose sanguínea e reduziu o percentual de hemoglobina glicada. Tais mudanças na glicemia foram notadas, a partir da quarta semana de execução do treinamento físico.

As mudanças nos parâmetros glicêmicos não foram notadas em apenas quatro semanas de treinamento, do estudo de Avery et al. ${ }^{21}$, uma vez que não apresentou diferenças nos níveis de glicose sanguínea, hemoglobina glicada, incidência de terapia com insulina, mudanças no peso materno e do recémnascido e hipoglicemia ao nascer, mesmo apresentando melhora significativa da capacidade cardiorrespiratória, e essa estar relacionada à melhora da captação de insulina e consequentemente melhora do controle glicêmico. Talvez, se o treinamento fosse prolongado por algumas semanas o comportamento parâmetros glicêmicos pudessem ser diferentes.

Ainda relacionando o exercício aeróbico à obesidade materna, este ajuda a limitar o ganho de peso durante a gestação, fato este que pode resultar em menor retenção de peso pós-parto, e assim diminuir a incidência de diabetes tipo 2 e outras comorbidades relacionadas ao ganho de peso e obesidade ${ }^{31}$. Além disso, inserir o exercício à rotina diária durante a gestação pode resultar em mudança permanente no estilo de vida. As mudanças no estilo de vida devem ser orientadas visando à prevenção do diabetes tipo 2 , visto que o exercício e a redução de peso são considerados essenciais na prevenção e manejo dessa doença ${ }^{13}$. No estudo de Artal et al. ${ }^{22}$, as participantes que perderam ou mantiveram o peso, demonstraram uma tendência a apresentar menos desfechos adversos para o feto, principalmente com relação à macrossomia fetal. Devido ao tamanho da amostra ser pequeno, esses achados não possuem significância estatística, apenas sugerem que desfechos favoráveis relacionados ao feto ocorrem devido ao menor ganho de peso durante a gestação.

Os estudos apresentados na presente revisão investigaram os efeitos crônicos do treinamento aeróbico no tratamento do DMG e demonstraram que a execução de um programa de exercício físico durante a gestação foi seguro, uma vez que não houve diferenças significativas entre os grupos que realizaram treinamento físico e seus respectivos controles com relação às complicações maternas e fetais. Mesmo os programas de exercícios parcialmente supervisionados apresentaram-se seguros para as gestantes com $\mathrm{DMG}^{19-22}$.

Contudo, o fato do treinamento ser realizado sem supervisão integral pode ter prejudicado os resultados desses estudos, como no estudo de Avery et al. ${ }^{21}$, as participantes relataram que a intensidade do exercício domiciliar era menor que quando realizado de maneira supervisionada, se esse fato tivesse sido evitado, através de supervisão em todas as sessões, as gestantes poderiam ter apresentado melhora do controle glicêmico. E no estudo de Artal et al. ${ }^{22}$, a aderência do grupo dieta e exercício foi $50 \%$ com relação ao programa de exercício, talvez se houvesse supervisão poderia ter sido maior. Realizar um exercício físico com supervisão de um profissional especializado pode garantir, uma correta execução, um maior controle da intensidade e através desses fatores uma maior segurança, além disso, os profissionais motivam o indivíduo a realizar o exercício diminuindo assim as chances de desistência. 


\section{Efeito crônico do treinamento de força e o DMG}

Um número limitado de estudos verificou o papel do treinamento de força realizado por gestantes com DMG. Alguns autores justificaram a utilização desse tipo de treinamento, uma vez que os exercícios aeróbicos podem se tornar desconfortáveis no decorrer da gestação, e que os exercícios de força utilizando, principalmente, os membros superiores são mais confortáveis nesse período, além de apresentarem pequeno estresse mecânico no tronco e assim não estimularem contrações uterinas ${ }^{23}$. Os trabalhos utilizando treinamento de força, apesar de similares em sua prescrição de treinamento, apresentaram resultados diferentes. Barros et al..$^{24}$, concluíram que o exercício foi efetivo para mulheres com DMG, visto que reduziu o número de pacientes que necessitaram de insulina e melhorou o controle glicêmico nessa população. Porém, no estudo de Brankston et al. ${ }^{23}$, os autores não encontraram diferença significativa no número de pacientes que apresentaram prescrição de insulina entre os grupos exercício e o grupo controle, no entanto, demonstraram efeito benéfico da prática de exercício de força, uma vez que a quantidade de insulina prescrita foi menor no grupo dieta e exercício, assim como o tempo de latência maior entre a primeira visita e o início do uso de insulina.

As peculiaridades presentes em cada estudo parecem ter sido responsáveis pelas diferenças nos seus resultados. Observa-se que Barros et al. ${ }^{24}$ não utilizaram critérios de inclusão e exclusão muito rigorosos ao selecionarem sua amostra, visto que foram incluídas participantes em diferentes semanas gestacionais e com diferentes índices de massa corporal, com isso seu número de participantes foi duas vezes maior do que no estudo de Brankston et al. ${ }^{23}$ Os programas de exercício também apresentaram diferenças, visto que Barros et al. ${ }^{24}$ monitoraram semanalmente seu programa, adequaram o exercício à intensidade, corrigiram a execução do exercício e modificaram o comprimento da banda elástica, ou seja, conforme a evolução do treinamento das gestantes, o programa foi evoluindo, garantindo a possibilidade de novas adaptações. Diferentemente de Brankston et $\mathrm{al}^{23}$, no qual a progressão foi realizada sem um monitoramento da evolução das participantes.

Ambos os trabalhos apresentaram um nível de aderência satisfatório, com poucas perdas, o que foi atribuído ao fato do exercício ter sido realizado em casa. Contudo, Brankston et al. ${ }^{23}$ relataram que devido ao programa de exercício ter sido parcialmente supervisionado, algumas participantes não realizaram o treinamento três vezes na semana, conforme fora indicado, e, provavelmente, se elas realizassem nessa frequência, um maior número de gestantes poderia ter evitado o uso de insulina. Segundo os estudos que apresentaram os efeitos do treinamento de força no DMG, esse tipo de treinamento parece não apresentar efeitos adversos durante a gestação e no momento do parto, com isso, a prática de um programa de exercício de força no DMG demonstrou-se segura tanto para mãe quanto para o feto ${ }^{23,24}$.

A presente revisão sistemática visou a abordar os aspectos relacionados aos principais efeitos do exercício físico no tratamento do DMG. Pode-se observar que existem poucos estudos relacionados aos efeitos do treinamento aeróbico e de força para manejo do DMG, contudo, a maior parte deles demonstrou que o exercício é capaz beneficiar as gestantes que o praticaram, sendo seguro e efetivo para controlar a glicemia, e em alguns reduzir a necessidade de insulina. 
O exercício aeróbico foi o escolhido na maioria dos trabalhos publicados, o que pode ser devido as suas facilidades, uma vez não há necessidade de grandes recursos e suas modalidades são extremamente funcionais, tornando-o prático, de baixo custo e reprodutível. Entretanto, os estudos que utilizaram treinamento resistido alcançaram seu objetivo de controle glicêmico, levando a crer que o exercício resistido também é bem indicado. Treinamentos aeróbicos e de força, com três sessões semanais, variando entre 20 minutos e 45 minutos e com intensidade moderada $\left(50 \%\right.$ a $70 \% \mathrm{VO}_{2 \text { máx }}$ ou percepção subjetiva de esforço) foram os que apresentaram melhores resultados para o controle glicêmico. Percebeu-se que, quando o exercício não é supervisionado de maneira integral, algumas informações podem ser perdidas ou alteradas gerando viés ao estudo.

Outra vantagem da realização de um programa de exercícios durante a gestação, é que as mudanças para hábitos de vida mais saudáveis, com o objetivo de diminuição das necessidades de insulina e melhora do controle glicêmico, podem incentivar esses indivíduos a adquirir esses hábitos após a gestação, o que pode resultar em redução das chances de desenvolver diabetes tipo 2 ao longo da vida e também do desenvolvimento do DMG durante outra gestação ${ }^{31}$.

Considerando os achados dos estudos incluídos na presente revisão, é relevante, seguro e aconselhável incluir um programa de treinamento físico tanto aeróbico quanto de força como parte do tratamento do DMG. Todavia, tornase necessário a realização de novos estudos que verifiquem os efeitos de diferentes modalidades de exercício de maneira supervisionada, também seriam interessantes estudos que comparassem os efeitos do treinamento de força e do treinamento aeróbico. Assim como devem ser desenvolvidos estudos que envolvam o treinamento combinado, uma vez que esse tem se apresentado benéfico no manejo do diabetes tipo $2^{32}$. E baseado nos achados das pesquisas, criar um consenso sobre o tipo, a duração, a intensidade, e a frequência ideais do exercício físico para manejo do DMG.

\section{REFERÊNCIAS}

1. American Diabetes Association. Gestational diabetes mellitus. Diabetes Care. 2004;27:S88-90.

2. American Diabetes Association. Diagnosis and classification of Diabetes Mellitus. Diabetes Care. 2014;37:S81-90.

3. American Diabetes Association. Standards of Medical Care in Diabetes. Diabetes Care. 2011;34:S11-61.

4. Hunt KJ, Shuller KL. The increasing prevalence of diabetes in pregnancy. Obstetric and Gynecology Clinics of North America. 2007; 34:173-99.

5. American Diabetes Association. Diagnosis and classification of diabetes mellitus. Diabetes Care. 2011;34:S1-201.

6. American College of Obstetricians and Gynecologists. Gestational Diabetes. Practice Bulletin. 2001 no 30.

7. Mitanchez D, Burguet A, Simmeoni U. Infants Born to mothers with gestational diabetes mellitus: mild neonatal effects, a long-term threat to global health. The J of pediatrics. 2014;164(3):445-50.

8. Mottola MF. The role of exercise in the prevention and treatment of gestational diabetes mellitus. Curr Diab Rep. 2008;8:299-304.

9. Kim C, Newton KM, Knoop RH. Gestational diabetes and incidence of type 2 diabetes. Diab Care. 2002;25:1862-8.

10. Canadian Diabetes Association Clinical Practice Guidelines Experts Committee. Diabetes and Pregnancy. Can J Diabetes. 2013;37:S168-83. 
11. American Diabetes Association. Gestational diabetes mellitus. Diabetes Care. 2003;26 Suppl 1:103-5.

12. Weinert LS, Silveira SP, Oppermann ML, Salazar CC, Simionato BM, Siebeneichler A, et al. Diabetes gestacional: um algoritmo de tratamento multidisciplinar. Arq Bras Endocrinol Metab. 2011;55:435-45.

13. Artal R. Exercise: The alternative therapeutic intervention for gestational diabetes. Clin Obstet Gynecol. 2003;46:479-87.

14. Golbidi S, Laher I. Potential mechanisms of exercise in gestational diabetes. J Nutr Metab. 2013:1-16.

15. Colberg SR, Sigal RJ, Fernhall B, Regensteiner JG, Blissmer BJ, Rubin RR, et al. Exercise and type 2 diabetes. The American College of Sports Medicine and the American Diabetes Association: joint position statement executive summary. Diabetes Care. 2010;33:2692-6.

16. Tobias DK, Zhang C, van Dam RM, Bowers K, Hu FB. Physical activity before and during pregnancy and risk of gestational diabetes mellitus. Diabetes Care. 2011;34:223-9.

17. Canadian Diabetes Association. Clinical practice guidelines for the prevention and management of diabetes in Canada. Can J Diabetes. 2008;32:S1-201.

18. Mottola MF, Giroux I, Gratton R, Hammond JA, Hanley A, Harris S, et al. Nutrition and exercise prevent excess weight gain in overweight pregnant women. Med Sci Sports Exerc. 2010:265-72.

19. Jovanovic-Peterson L, Durak EP, Peterson CM. Randomized trial of diet versus diet plus cardiovascular conditioning on glucose levels in gestational diabetes. Am J Obstet Gynecol. 1989; 161:415-9.

20. Bung P, Artal R, Khodiguian N, Kjos S. Exercise in gestational diabetes. No optional therapeutic approach? Diabetes. 1991;40:182-5.

21. Avery MD, Leon AS, Kopher RA. Effects of a partially home-based exercise program for women with gestational diabetes. Obstet Gynecol. 1997;89:10-5.

22. Artal R, Catanzaro RB, Gavard JA, Mostello DJ, Friganza JC. A lifestyle intervention of weight-gain restriction: diet and exercise in obese women with gestational diabetes mellitus. Appl Physiol Nutr Metab. 2007;32:596-601.

23. Brankston GN, Mitchell BF, Ryan EA, Okun NB. Resistance exercise decreases the need for insulin in overweight women with gestational diabetes mellitus. Am J Obstet Gynecol. 2004;190:188-93.

24. Barros MC, Lopes MAB, Francisco RPV, Sapienza AD, Zugaib M. Resistance exercise and glycemic control in women with gestational diabetes mellitus. Am J Obstet \& Gynecol. 2010;203:551-6.

25. Borg G. Borg's perceived exertion and pain scales. Champaign, IL: Human Kinetics; 1998.

26. Kühl C. Etiology and pathogenesis of gestational diabetes. Diabetes Care 1998;21:B19-B26.

27. Ruchat S, Mottola MF. The important role of physical activity in prevention and management of gestational diabetes mellitus. Diabetes Metab Res and Rev. 2013;29:334-46.

28. Buchanan TA, Xiang AH. Gestational diabetes mellitus. Clin Invest. 2005;115:485-91.

29. Xiang AH, Peters RK, Trigo E, Kjos SL, Lee WP, Buchanan TA. Multiple metabolic defects during late pregnancy in women at high risk for type 2 diabetes mellitus. Diabetes. 1999;48:848-54.

30. Deirdre TK, Zhang C, Dam RM, Bowers K, Hu FB. Physical activity before and during pregnancy and risk of gestational diabetes mellitus: a Meta-analysis. Diabetes Care. 2011;34:223-9.

31. Catalano PM, McIntyre HD, Cruickshank JK, McCance DR, Dyer AR, Metzger BE, et al. The Hyperglycemia and Adverse Pregnancy Outcome Study; associations of GDM and obesity with pregnancy outcomes. Diabetes Care. 2012;35:780-6.

32. Umpierre D, Ribeiro PB, Kramer CK, Leitão CB, Zucatti AT, Azevedo MJ, et al. Physical activity advice only or structured exercise training and association with HbA1clevels in type 2 diabetes. JAMA. 2011;305:1790-9.

ENDEREÇO PARA

CORRESPONDÊNCIA

ELISA GOUVÊA PORTELLA

Rua Felizardo, 750 - LAPEX sala 208

Jardim Botânico

CEP 90690-200 - Porto Alegre/RS

Telefone: 5551 3308-5820

E-mail: elisaportella_rglayahoo.com.br 